Noname manuscript No.

(will be inserted by the editor)

\title{
A Digital Image Correlation algorithm with light reflection
} compensation

\author{
Martin Poncelet • Hugo Leclerc
}

Received: date / Accepted: date

\begin{abstract}
This work focuses on the influence of light reflection on Digital Images Correlation results at the macroscopic scale, and on a way to circumvent this problem. It shows that the local displacement uncertainty rises up to 5 times the usual one when a reflection occurs. An observation of the topography of the speckle reveals an important sub-pixel roughness that explains the grey level fluctuations at the pixel scale, spoiling the calculation the gradient of the texture. To circumvent this problem, a new DIC algorithm is proposed, based on a single minimization with several pairs of images where the reflections are located in different regions. For each image, weighting functions are used to 'exclude' the reflection regions from the calculation, while the necessary information is obtained from the other images.
\end{abstract}

M. Poncelet

Laboratoire de Mécanique et Technologie (LMT-Cachan), ENS de Cachan/CNRS UMR 8535/Univ. Paris 6/PRES UniverSud Paris, 61 Avenue du Président Wilson, F-94235 Cachan

Cedex, France E-mail: poncelet@lmt.ens-cachan.fr

H. Leclerc

Laboratoire de Mécanique et Technologie (LMT-Cachan), ENS de Cachan/CNRS UMR 8535/Univ. Paris 6/PRES UniverSud Paris, 61 Avenue du Président Wilson, F-94235 Cachan Cedex, France E-mail: leclerc@lmt.ens-cachan.fr 
Keywords Full field measurement · Digital Image Correlation · Light reflection ·

Speckle · Uncertainty assessment

\section{Introduction}

The increasing performances of optical full-field kinematic measurement techniques has lead to their widespread use. Among the many possible ones [1], such as photoelasticity, moiré, holographic and speckle interferometry, grid method and Digital Image Correlation (DIC), this last one [2] is very often chosen for its 'simple' use conditions in comparison with the other techniques. Consequently, DIC measurements are now performed out of the research laboratories [3], where the testing conditions are no longer optimal, or performed on industrial products which surfaces are no longer flat $[4,5]$. With these new configurations, lightning problems appear: non-constant illumination, cast, coreshadows and reflections.

Non-constant global illumination issues may be prevented by cunning protocols: Yoneyama et al. [3] performs tests on a bridge at night with artificial illumination, Grant et al. [6] uses a combination of illumination and optical filter at the same wavelength to prevent disturbances.

Large scale heterogeneity of illumination issues due to reflection can be partially solved during measure by use of polarized filters [7]. However every reflection can not be withdraw with this technique, so that post-processing techniques are necessary. One may want to 'enhance' images with several existing techniques, virtually lighting up shadows or correcting burned areas. Based on empirical or scientific models, some techniques aim aesthetic or visibility purposes $[8,9]$, while others aim quantitative purposes, like automatic detection [10]. In any case, these visual enhancements are not of interest from a DIC point of view, potentially introducing spurious grey level gradients that spoil the results. Consequently, some full-field measurement algorithms consider an optical flow conservation relaxation. Local DIC algorithms use normalized 
cross-correlation calculation for each sub-image for example $[12,13]$, while global DIC algorithms use a local grey level correction at the scale of the analyzed elements [14]. To go further with this idea, it is possible to use the variation of the optical flow as a pertinent information in itself, for example to measure both kinematic and thermal fields from IR images [15].

Dealing with shadows is more difficult. The best theoretical solution to light up shadowy areas is the use a homogeneous light source like dome lights, but it is experimentally impossible in many cases. Several light sources are then often preferred, but it multiplies the number of unwanted reflections. Reflections are thus nearly unavoidable in the case of complex shapes.

Because of the strong variations due to reflections, images are often characterized by very low grey levels, near saturation or saturation situations. The saturated areas do not carry information, so that the measurement is strictly impossible. A simple way to prevent this is to use a slightly higher dynamic range (for example a semi-professionnal Digital Single Lens Reflex camera with up to 14 bit depth), or High Dynamic Range devices (scientific grade cameras with more than 16 bit depth and very low noise) during the experiment.

The remaining important issue is that during the translation of the object, the texture of the surface follows it while the shadows and reflections do not exactly (Fig. 1). During a rotation the texture may nearly not move from the camera point of view near the rotation axis, while reflections change tremendously. Shadows may also evolve, usually only slightly but non-convex or partially flat objects can present sudden form and cast shadows. If an adequate lightning setup is used, the difficulties linked to shadows may be suppressed as stated before, but reflections still exists and are generally characterized by high grey level gradients. Consequently their gradients may be 'misinterpreted' as a texture displacement. 

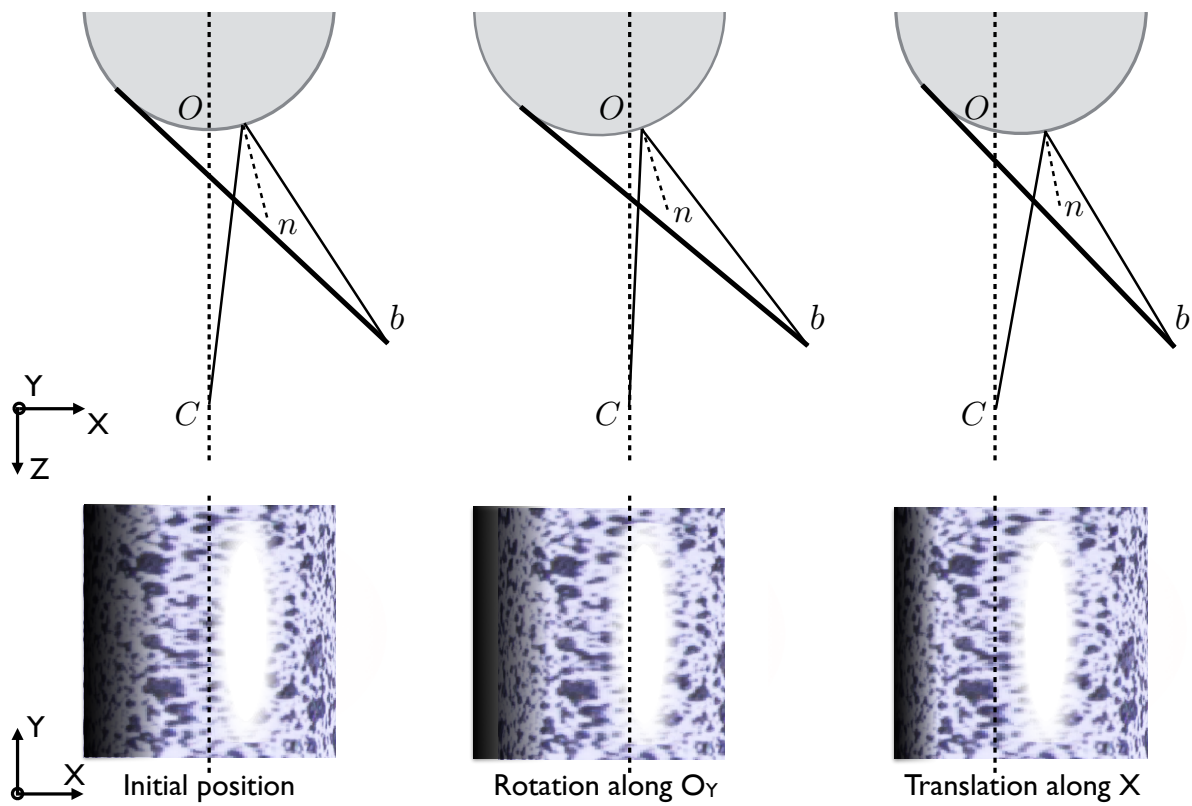

Fig. 1 Artificial example of evolution of the reflection and shadow positions in the case of a rotation and a translation of a cylinder. First, the apparent displacement near the rotation axis is nearly zero, while the reflection get closer to it. Second, the reflection apparent displacement is smaller than the real translation of the object.

The simplest way to circumvent this problem afterward is to exclude the problematic areas from the measurement zone. The information lost in the disturbed areas may be important, all the more if stereovision techniques (like stereo-DIC $[13,16]$ ) are used, because a reflection may affect a different zone on each picture, contrary to shadows.

A second problem is related to the fluctuations of the apparent texture, due to changes of the angles between the lights, the cameras and the surfaces. They may lead to local additional uncertainty which should be taken into account by a local weighting. Up to now, most full-field kinematic measurements [17] use an homogeneous weight, assuming a constant apparent noise.

The present paper proposes to study the influence of reflections on DIC measurements and then to develop a 'reflection-proof' DIC technique. One focuses on the case of a DIC measurement at the 'macroscopic scale' with a regular, white-light, as in many 
industrial and academic applications. The study uses regular speckles obtained with mat sprays and no satured areas to quantify the measurement quality loss. The results must therefore be considered as obtained 'in the best measurement conditions' (no gloss paint, no saturated areas). It will be shown that it is not possible to correct these reflections by numerically taking into account the geometry of the specimen because of the inner characteristics of the coating, which induce unpredictable reflections at the pixel scale.

The proposed 'reflection-proof' technique requires a simple experimental setup and is adapted to non-satured cases as well as satured ones. It is based on the use of a quick succession of different light sources: several different light sources sequentially illuminate the surface during a negligible short time in comparison with the object velocity. This technique enables to exploit any area of the studied object since the reflections appear at different locations on the images while the mechanical configuration is the same. A dedicated algorithm solves a global problem, i.e. takes into account all the images corresponding to the different light sources, with an appropriate local weighting of each image. A validation of this technique will be last shown in the case of a sphere illuminated by 2 light sources, and observed by a single camera.

The paper is divided as follow. In a first part, the influence of the reflection on DIC measures is studied: a setup is proposed to study the influence, the results are presented for a standard algorithm and one with a local grey level correction, and then the reasons of this influence are searched with a measurement of the evolution of the grey level with the angle of the incident light, and a sub-pixel scale roughness study of the coating. In a second part, the reflection-proof technique, called 'Multiple Illumination Digital Image Correction' is explained and mathematically formulated. A third part presents the validation of the technique, and a discussion follows. 
2 Light reflection on a typical paint speckle

2.1 Experimental setup and protocol

To characterize the reflection of light on a painted speckle, the optical bench presented

Fig. 2 and 3 is used. All the devices and paints used thereafter are similar to the ones generally used for DIC measurement. The only special feature of the bench is the possibility to accurately position every devices. Its principe is to observe an object with a camera, while the angle of the object $\alpha_{c}$ (and so the angle of the incident light $\alpha_{i}$ used to illuminate the object) is changed by use of rotation stages. The camera is a Canon EOS 60D Digital Single Lens Reflex equipped with $60 \mathrm{~mm}$ focal lens. The image has a resolution equal to $5184 \times 3456$ pixels, and is saved in a lossless format (.raw). The white light source (EFFISHARP NearField 000-02 LED lamp, Effilux, Orsay (France)) is considered as point-wise and homogeneous. The observed objects are respectively a 50x75-mm polished aluminum plate and a 82.5 - $\mathrm{mm}$ steel sphere covered with a white layer of mate paint and then a spray of black mate paint (RAL 9010 'Noir pur mat', RAL 9010 'Blanc pur mat', Ront Production, France). The optical axis of the camera is in the same horizontal plane as the centre of the sphere, while lightning $b_{1}$ (reps. $b_{2}$ ) is slightly above (resp. below).

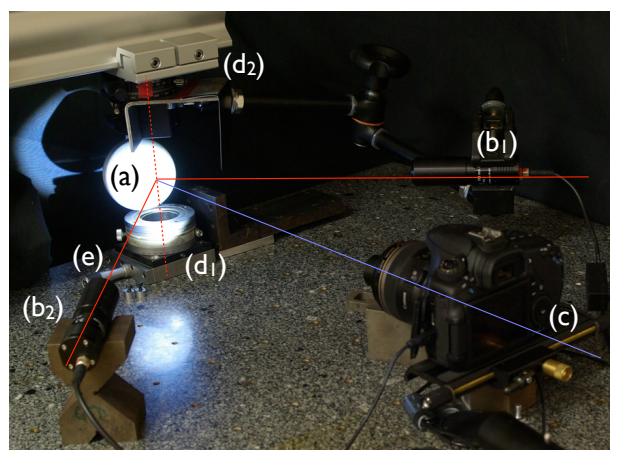

Fig. 2 Optical setup: (a) object, ( $\left.b_{1}\right)$ light source, $\left(b_{2}\right)$ additional light source used in section 3.3, (c) DSLR, $\left(\mathrm{d}_{1}\right)$ rotation stage for objet tilt, $\left(\mathrm{d}_{2}\right)$ used rotation stage for light tilt, (e) translation stage. 


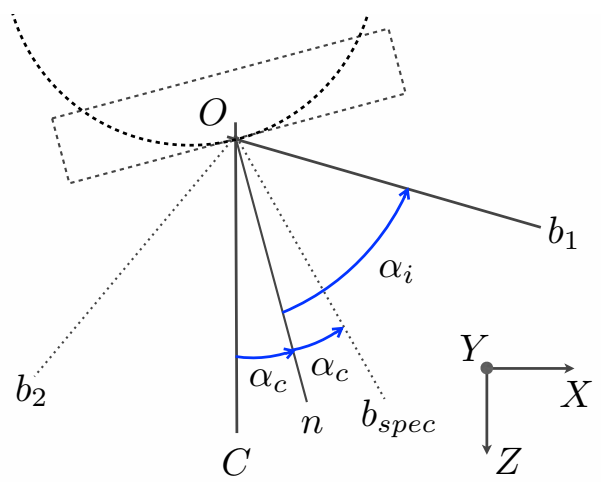

Fig. 3 Scheme of the setup with sphere and plane plotted in dashed lines. $O C$ is the optical axis of the camera aligned with $Z$ axis, with its focal plan parallel to $X Y$. On is the normal to the surface of the object at the intersection with $O C$, and $O b_{1}$ the incident light. The rotation stages axes are aligned with $Y$ and pass through point $O$.

2.2 Light reflection influence on kinematic measurement

To study the reflection influence on the DIC measure, the sphere is placed on the rotation stage with $\alpha_{c}=0$ at the beginning of the test. In this configuration, the rotation axis is considered on the point of the sphere closest to the camera (Fig. 3). Light source $b_{1}$ is on, and $\alpha_{c}+\alpha_{i}$ is constant during the test (i.e. the light is fixed with respect to the camera). The aperture is so that the observed surface is in the depth of field. The exposure time is so that no pixel is saturated (the difference between the maximum grey level value and the saturation value (65535) is around 60, i.e. around a thousandth of the dynamic range). Images are taken for different $\alpha_{c}$, ranging from 0 to $14^{\circ}$. Five $50 \times 100$ pixels areas noted $A 0$ to $A 4$ are chosen along the horizontal axis $X$ (Fig. 4). The reflection displacement is clearly visible between the initial position in area $A 4$ (Fig. 4 (a)) and the last one in area $A 2$ (Fig. 4 (b)). The value of the grey level (in percent of the total dynamic range) along the horizontal line crossing the disturbed area $f_{\text {line }}(x)$ is plotted to better show the displacement of the reflection. 
DIC measurement is first done with a TRI3 'finite element' like algorithm [18, 19]. The reference image is the one at $\alpha_{c}=0$, while deformed images correspond to the different other $\alpha_{c}$ values. The mesh size, as for the next algorithm results, is 25 pixels. The residual is calculated for each deformed image as the standard deviation of the difference between the reference image and the deformed image corrected by the found displacement using a bicubic interpolation for the sub-pixel displacement. The average residual for each image is around $2 \%$ of the effective dynamic range (i.e. maximum minus minimum grey level values of the images), which proves that the calculation has converged (usual mean residual without reflection is about $2 \%$ ). Fig. 5 (a) shows the measured displacement $U_{y}$ along $Y$ for a rotation equal to $0^{\circ} 20^{\prime}$. This displacement along $Y$ (parallel to the rotation axis) is due to the perspective effect. It is presented here instead of the displacement along $X$ because it has a smaller magnitude, so that the perturbations are comparatively more visible. Even though the general trend is satisfactory, a slight noisy area is noticeable. It corresponds to the region $A 4$ of the $0^{\circ}$ image depicted Fig. 4 (a) where the reflection occurs. The displacement along $Y$ corresponding to a $14^{\circ}$ rotation is presented Fig. 5 (b). The magnitude of the displacement is far more important, so that the previous noisy area is no longer directly visible. Since one may be interested in displacement heterogeneities with small magnitude embedded in a large quasi-uniform displacement (for example due to a rigid body motion), it is proposed thereafter to subtract the long wave components of the displacement field to better understand the reflection disturbance.

To quantitatively assess the perturbation of the displacement field due to the reflection, the 5 areas will be specifically studied. A high-pass filtered field $\widetilde{U_{y}}$ is calculated by subtracting to $U_{y}$ a field obtained by applying a radial gaussian filter to $U_{y}$. The standard deviation of the gaussian filter is equal to 40 pixels, so that $\widetilde{U_{y}}$ does not contain the displacement corresponding to the rotation of the sphere and the integer locking artifacts ( [18]), but only keeps the high frequency heterogeneity of the 


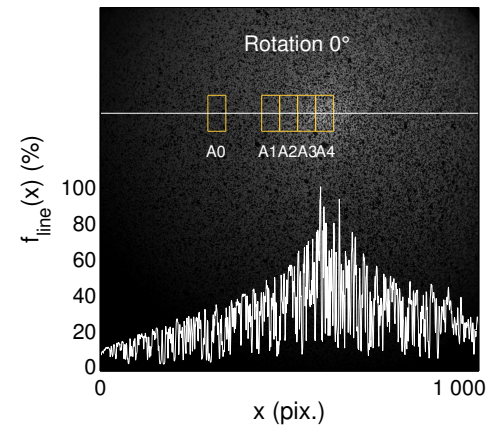

(a)

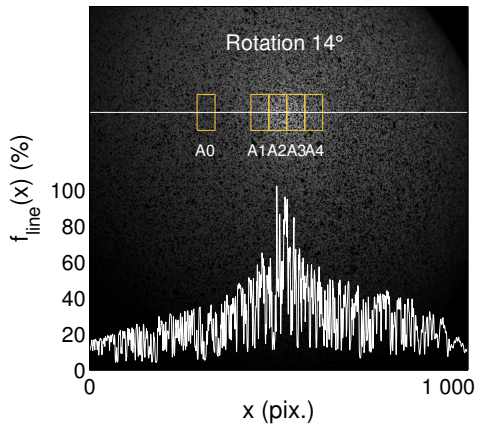

(b)

Fig. 4 Images of the sphere with the reflections for an angle equal to $0^{\circ}$ (a) and $14^{\circ}$ (b). The areas used for the perturbation assessment are represented by the yellow rectangles. The value of the grey level (in percent of the total dynamic range) along the white line crossing the disturbed area $f_{\text {line }}(x)$ is also plotted to better show the displacement of the reflection.

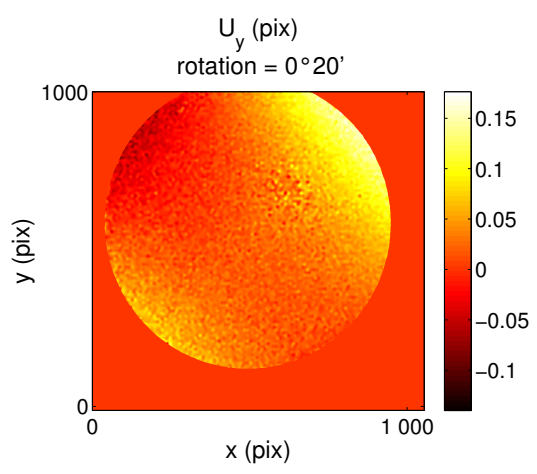

(a)

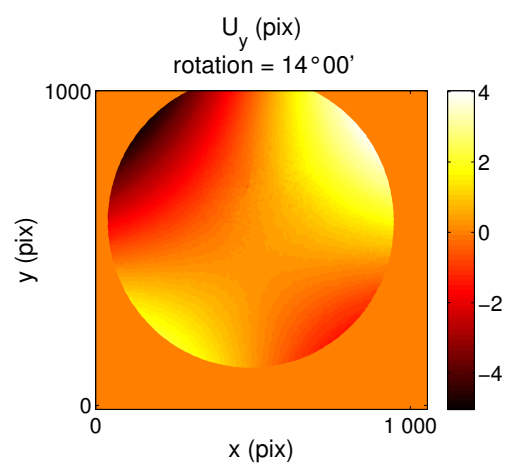

(b)

Fig. 5 Vertical displacement $U_{y}$ measured for a $0^{\circ} 20^{\prime}$ (a) and $14^{\circ}$ (b) rotation.

field in the studied areas. For each of the 5 areas, the mean value of $U_{y}$ is plotted Fig. 6 , showing a linear evolution with $\alpha_{c}$. The perturbation $P$ is calculated as the standard deviation of $\widetilde{U_{y}}$ on each area. $P$ is plotted as a function of the rotation angle on Fig. 7 (b). Three distinct behaviours are notable :

- For area $A 0$, the reflection is far away for any image, so that it is not affected.

The value of the perturbation is very low $\left(P \approx 4 \times 10^{-3}\right.$ pix $)$ for small angle values $\left(\leq 2^{\circ}\right)$ and increases slowly with the rotation (up to $P \approx 4 \times 10^{-2}$ pix). 
The perturbation is directly linked to the measurement uncertainty of the DIC technique.

- For area $A 4$, the perturbation increases up to 4 times higher because the reflection on the reference image $\left(0^{\circ}\right)$ is over this area. This 'additional' perturbation exists even for very small rotation angles $\left(<1^{\circ}\right)$ and is nearly constant once the reflexion is away from the area on the current image.

- For areas $A 1$ to $A 3$, the perturbation is nearly equal to $A 0$ one for small rotation angles because the reflection is then not over these areas. Nonetheless for higher rotation angles, the reflection 'crosses' the different areas, and $P$ increases up to 5 timesthe perturbation of area $A 0$. Once the reflection has exceeded the area, the perturbation decreases as shown for $A 2$ and $A 3$. Roughly speaking, for area A1, the perturbation only increases, since the area is lately reached by the reflection at a rotation angle equal to $\approx 12^{\circ}$. For these areas, the maximum value of the perturbation increases with the angle of reflection necessary to obtain it, i.e. a reflection due to a more important incident angle is more detrimental than a smaller one.

The measurement uncertainty is thus multiplied by a factor $\approx 4$ to $\approx 5$ if one compares the affected area and an unaffected one for the same angle value. The total increase of uncertainty when the worst result (area $A 1$ for $12^{\circ}$ ) is compared to the best one (area $A 0$ for $\left.0^{\circ} 20^{\prime}\right)$ is a factor around 25 .

A grey level correction is now added to the previous technique. It uses linear shape functions with the same mesh as for the kinematic quantities, as in [14]. This Grey Level Correction Digital Image Correlation algorithm (GLC-DIC) is used on the same set of images to assess to what extent such correction may improve the results. During the iterations, one computes a contrast and luminosity correction, using finite element representation, on the same mesh than the one that is used to evaluate the displacements. The obtained perturbation values are plotted on Fig. 8 . 


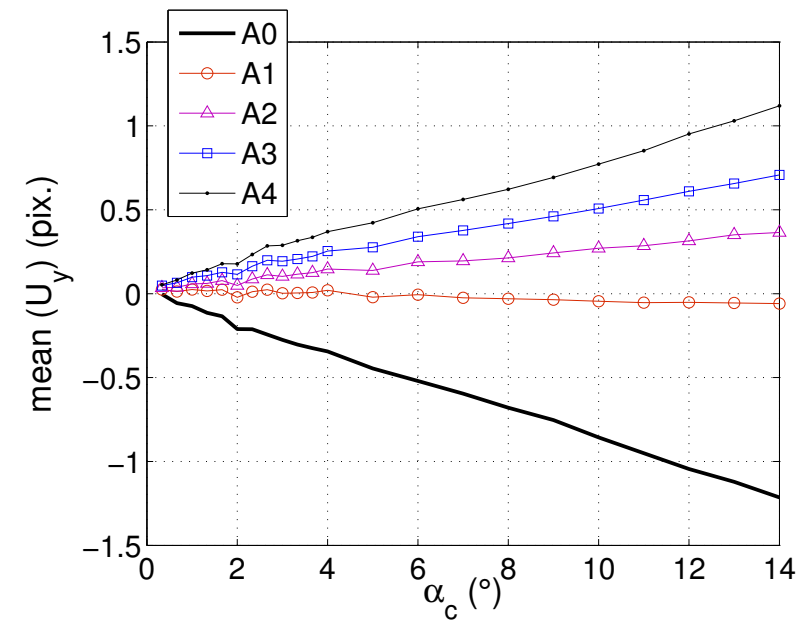

Fig. 6 Mean displacement for the different areas, as a function of the rotation angle of the object $\alpha_{c}$ in the case of a standard DIC algorithm.

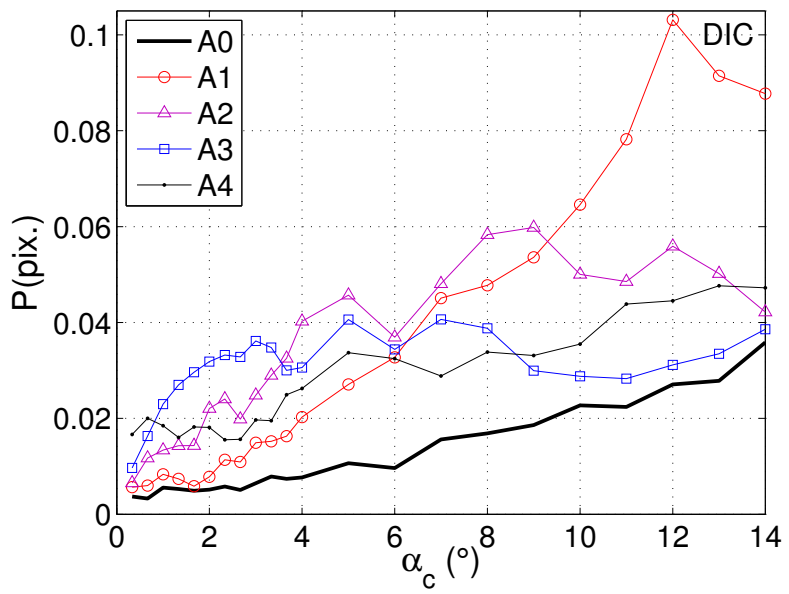

Fig. 7 Perturbation $P$ for the different areas, as a function of the rotation angle of the object $\alpha_{c}$ in the case of a standard DIC algorithm.

- For area $A 0$, the perturbation is slightly lower than with the standard algorithm.

- For areas $A 4$ and $A 3$, the perturbation is also lower, but the important increase occurring for small $\alpha_{c}$ values $\left(\leq 2^{\circ}\right)$ is nearly unchanged. 
- For areas $A 1$ to $A 2$, the maximum value is clearly less than with the standard algorithm, but it is still higher than for area $A 0$.

One concludes that the grey level correction is able to compensate for the global variation of illumination taking place during the large rotation of the sphere. However this linear grey level correction at the scale of the element is not adequate to delete the important increase of the perturbation for small rotation angles (i.e. the most common case during an experiment).

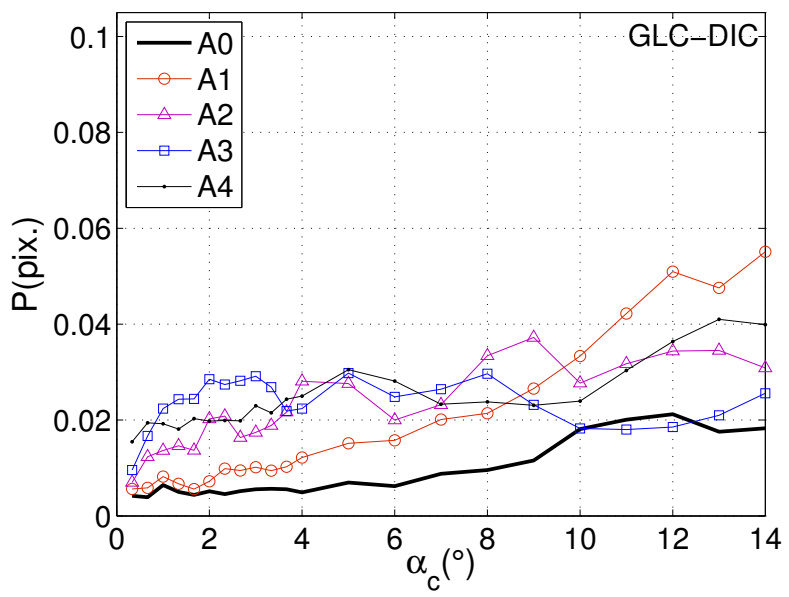

Fig. 8 Perturbation $P$ for the different areas, as a function of the rotation angle of the object $\alpha_{c}$ in the case of a DIC algorithm with grey level correction.

2.3 Grey level evolution with light orientation

The high effect of reflection on full field measurement results for large rotation angle was expected, since the grey levels evolve importantly. The smaller, but non negligible, effect for $\alpha_{c} \leq 2^{\circ}$ in the case of both DIC and GLC-DIC is less obvious because the reflection apparently does not change. To better understand the evolution of the grey 
level value in the disturbed area, the flat specimen is now used to study the evolution of the grey level with the angle of the incident light $\alpha_{i}$.

The specimen is placed on the rotation stage instead of the sphere (see dashed lines on Fig. 3), with the speckle plane on the rotation axis $O$. The normal axis $O n$ of the specimen is tilted with $\alpha_{c}=15^{\circ}$. The light source $b_{1}$ is now in the same horizontal $X Z$ plane as the optical axis. The angle $\alpha_{i}$ is then increased step by step with its own rotation stage with a picture taken for every position. The grey level value for 40 pixels located along the rotation axis $O$ is plotted as a function of $\alpha_{i}$ (Fig. 9).

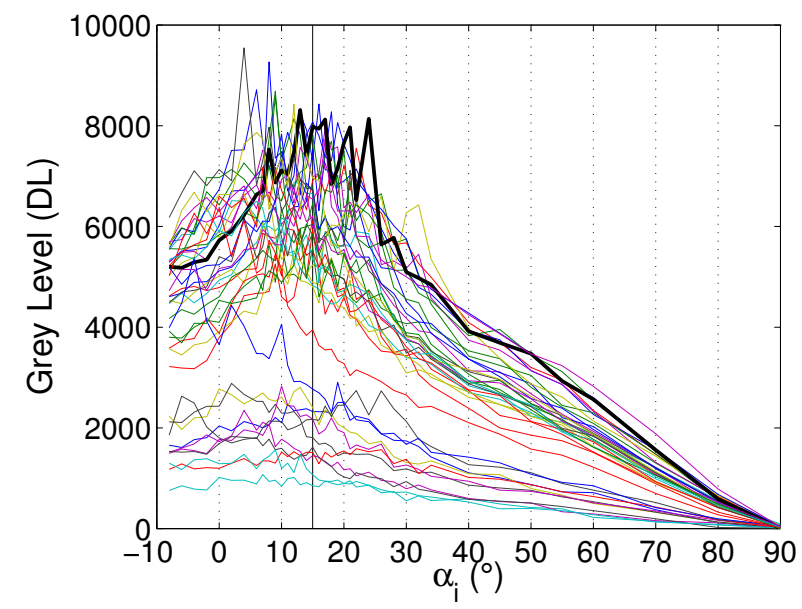

Fig. 9 Evolution of the grey level for several pixels located along the rotation axis $O$ as a function of the rotation angle $\alpha_{i}$.

For high values of $\alpha_{i}\left(90\right.$ to $\left.30^{\circ}\right)$ the evolution is smooth and corresponds to diffuse reflection, with total extinction at $90^{\circ}$. Two bundles of curves start from $90^{\circ}$, the upper one corresponding to the white background of the speckle (high reflectivity) and the lower one to the black dots (low reflectivity). For lower $\alpha_{i}$ values, the specular reflection contribution is visible. One expects a smooth increase with a maximum at $\alpha_{i}=15^{\circ}$ but the measure shows a very noisy evolution spreaded from roughly 30 to $0^{\circ}$. Consequently, one understands that it is no use to try to correct the reflection on the 
images with the sole knowledge of the optical configuration (theoretical geometry of the objet, relative positions of the camera and light source). The speckle itself induces a non trivial grey level evolution with the incident light angle.

2.4 Sub-pixel surface study

To understand the reason of this erratic evolution of the grey level value with the reflection, the roughness of the speckle of the flat specimen is studied. To measure it, a confocal chromatic sensor (130 $\mu \mathrm{m}$ range, $0.016 \mu \mathrm{m}$ uncertainty (standard deviation), CHR-150-L, STIL (France)) with a high precision translation stage $(0.1 \mu \mathrm{m}$ resolution, $1 \mu \mathrm{m}$ flatness defect over $100 \times 100 \mathrm{~mm}^{2}$, Micromesure 2, STIL (France)) is used. A $10 \times 10 \mathrm{~mm}^{2}$ area is measured on the flat specimen with a $5 \mu \mathrm{m}$ pitch and a mean over 5 consecutive measures. Both the intensity $I$ and the height $h$ can be analyzed afterward.

As a first approximation, the variation of height $h(x, y)$ (Fig. $10(\mathrm{a}))$ is related to the presence of black paint droplets as seen on the intensity map $I(x, y)$ (Fig. $10(\mathrm{~b}))$. This variation is less than $10 \mu \mathrm{m}$. The variation of the local slope $\arctan (|\nabla h|)$ on a $1 \times 1 \mathrm{~mm}^{2}$ region of Fig. 10 (a) is presented Fig. 11 (a). The uncertainty of the slope is evaluated to $0.23^{\circ}$ if one considers a $0.1 \mu \mathrm{m}$ uncertainty of $h$ measurement. The map is nonetheless very clear. The roughness is different on the white and black surfaces, probably due to the difference of filler or drying time (the black paint spray is dry in a few seconds while the white thick layer takes several minutes). In both case it reveals that the roughness is not random, but is characterized by a 'wrinkle' pattern with an average distance about $25 \mu \mathrm{m}$, i.e. at a lower (but not negligible) scale than the pixel physical size $(55 \mu \mathrm{m}$, represented by black lines on Fig. 11). The roughness is rather sharp (up to $15^{\circ}$ slope). This explains the high intensity variations in the reflection region for a small rotation in the previous experiment. One deduces that this effect can not be taken into account with an a priori knowledge of the theoretical surface of the object, 
as done for example in [10], where the high frequency fluctuations are not of interest. It can also not be withdrawn with techniques used for fringe projections - where the reflection has very low frequencies in comparison with the carrier frequency [11] because its spectrum is close to the one of the speckle. In other cases - object and speckle characteristic length several orders of magnitude over the roughness of the paint speckle, other speckle creation method, different light wavelength or image acquisition method - the conclusion may be different.

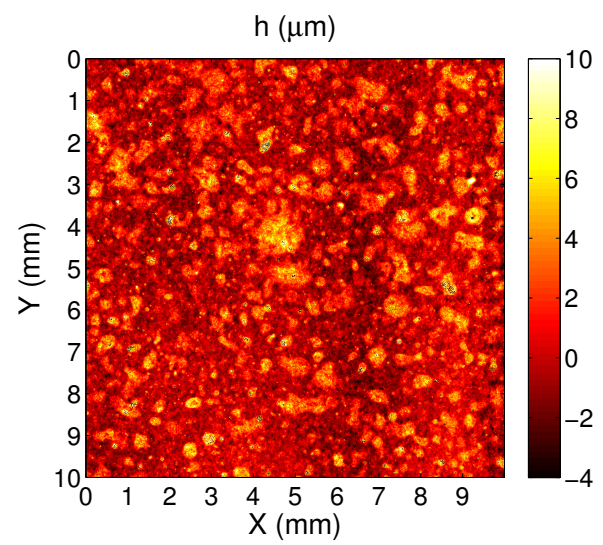

(a)

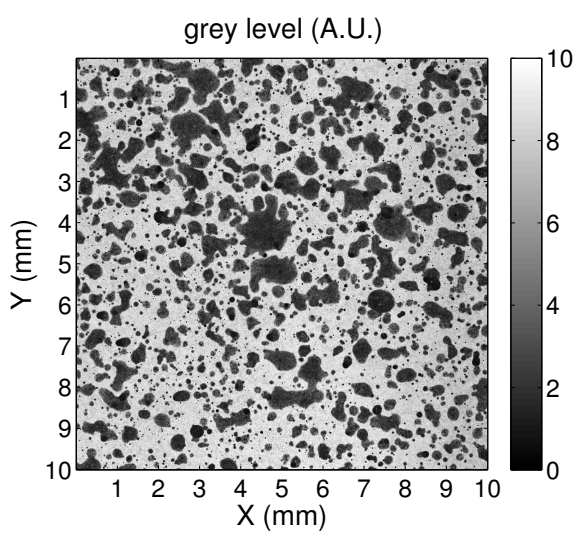

(b)

Fig. 10 Confocal chromatic measure of the speckle surface: (b) roughness (a 4-order bipolynomial fit have been subtracted to the raw measure) and (a) intensity map.

\section{Multiple Illuminations Digital Image Correlation}

\subsection{Principle}

The principle of the proposed method consists in calculating the data needed for the solving steps of each DIC procedure for each lighting condition, with weights allowing to exclude the regions where the reflection lies. By a choice of multiple illumination (Fig. 12), the different weights allow some kind of complementary contribution. A single 


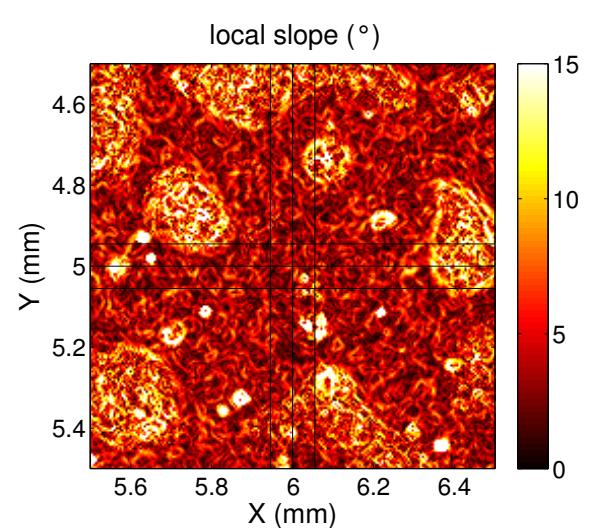

(a)

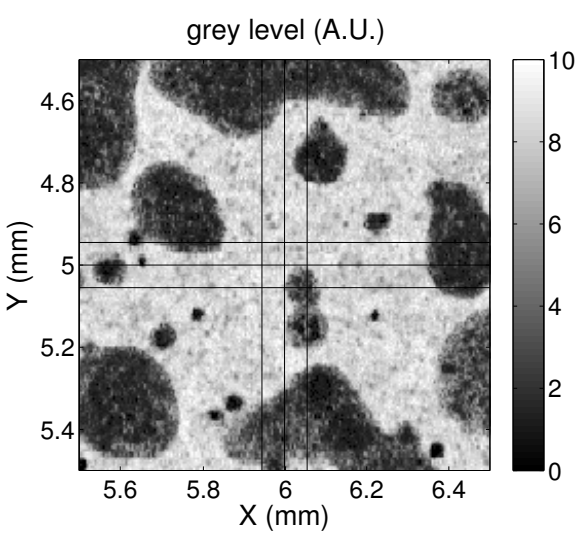

(b)

Fig. 11 Surface analysis: local slope (a) and Intensity map (b) on the same $1 \mathrm{~mm}^{2}$ area. Black lines stand for the pixel size.

global minimization procedure is then launched for each displacement field update iteration.

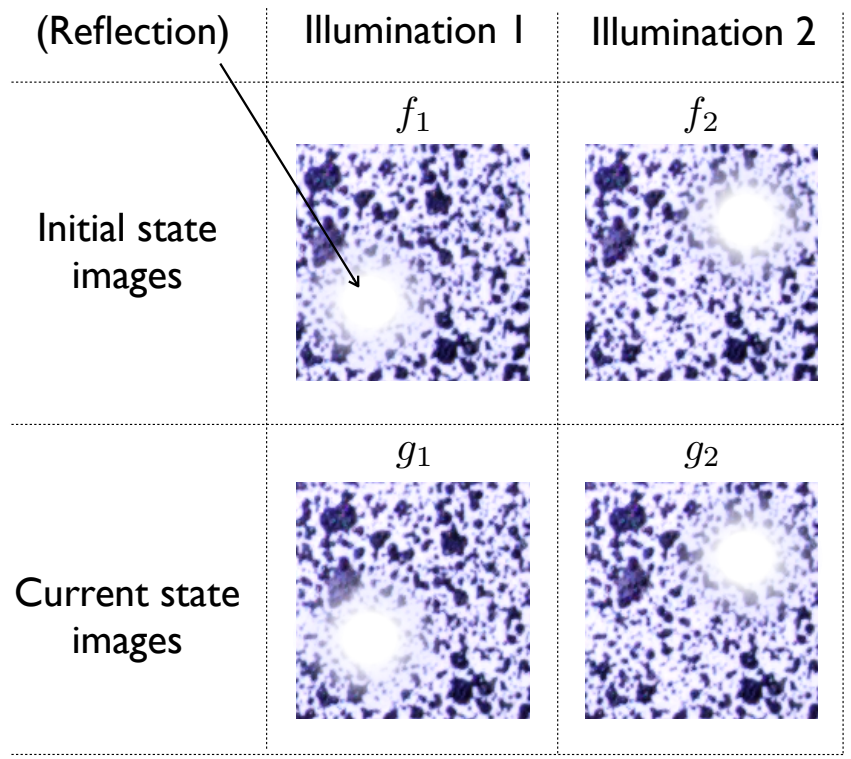

Fig. 12 Principle of multiple illuminations Digital Images Correlation. 
3.2 Mathematical formulation

In a DIC algorithm, an initial image $f$ is related to a deformed image $g$, the displacement field $\underline{u}$, and the camera noise $n$ by

$$
g(\underline{x}+\underline{u})=f(\underline{x})+n(\underline{x}) \quad .
$$

Solving the usual mono-illumination DIC problem, i.e. measuring $\underline{u}$, consists in minimizing a functional $\Phi$ over a set of possible displacements $\underline{v}$

$$
\Phi(\underline{v})=\iint(g(\underline{x}+\underline{v})-f(\underline{x}))^{2} d \underline{x} .
$$

In the present case, to take into account the fact that the same displacement is observed with different light sources (here 2), the functionnal is rewritten with two pairs of initial $\left(f_{1}, f_{2}\right)$ and deformed images $\left(g_{1}, g_{2}\right)$ as follow

$$
\begin{aligned}
\Phi(\underline{v})= & \iint\left(g_{1}(\underline{x}+\underline{v})-f_{1}(\underline{x})\right)^{2} p_{1}(\underline{x}) d \underline{x}+ \\
& \iint\left(g_{2}\left(\underline{x}+\underline{v}+\sum_{i} \beta_{i} \cdot \underline{\varphi_{i}}(\underline{x})\right)-f_{2}(\underline{x})\right)^{2} p_{2}(\underline{x}) d \underline{x}
\end{aligned}
$$

where $\beta_{i}$ are additional discrete unknowns, $\varphi_{i}$ are predefined rigid body motion functions, and $p_{1}$ and $p_{2}$ are two weighting functions.

If the $p_{j}$ functions are not used (or all of them set to a uniform value equal to 1), several pairs of images are used to assess the displacement field without respect of the existence of disturbed areas. Such a calculation leads to the results presented Fig. 13: the perturbation is nearly equal to the one obtained with one pair of images, only slightly lower. These functions are thus mandatory to delete the influence of the disturbed areas on both the reference image and the deformed image by taking into account the fact that under non-uniform lightning conditions (i.e. realistic conditions), some regions might not check the optical flow conservation. 


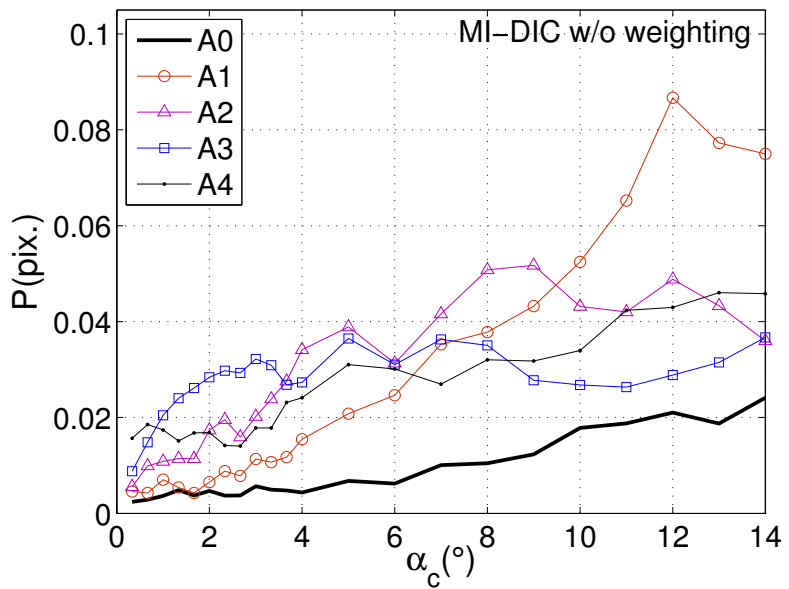

Fig. 13 Perturbation $P$ for the different areas, as a function of the rotation angle, with the proposed algorithm and no weighting.

A first method to calculate the $p_{j}$ functions needs the knowledge of the geometry of the setup (specimen as well as lightnings and camera, and of course the motion of the specimen) and the bidirectional reflectance distribution function (BRDF) corresponding to the speckle. It is then easy to define the theoretical disturbed areas (roughly corresponding to the region between $+/-15$ centered on the specular reflection on Fig. 9) prior to the MIDIC calculation. Unfortunately the geometry is not always known (no CAD of the setup, no stereo DIC to measure the displacement of the surface of the specimen). The BRDF is also rarely available. One may use the informations given in Fig. 9 to model the BRDF as a first approximation, but the measurement was restricted to one direction and only the incident angle has been probed. That would probably leading to a poorly relevant BRDF model.

A second method, more arbitrary but relevant, is to define the area that is disturbed (for example by using a criterion like the standard deviation of the displacement being over a given threshold of the usual standard deviation found on an unaffected area) after a first standard DIC calculation on the first pair of deformed images. Once the area has been assessed, one measures the corresponding average grey level on the perimeter of 
this area on the corresponding blurred images and uses it as a threshold to define the weighting functions for every images. This method has to be tuned for every different setup, but it is simple, fast and does not require to know the BDRF and geometry, so that it is presented thereafter.

We will only consider binary weighting functions, computed with the simple combination of a blur filter and a threshold. These weighting functions will merely suppress the regions where the light reflections may induce difficulties. For every angle value, one has first to blur the images taken with each light source to compute the weighting functions. A 2D isotropic filter is chosen, with a radius set to 256 pixels in the Fourier space, i.e. $1 / 4$ of the image size. It thus only keeps the average grey level evolution due to the lightning, and not due to the texture itself. An example is given for the angle equal to $0^{\circ} 20^{\prime}$ and the $b 1$ lightning on Fig. 16, where (a) represents the image and (b) the blurred image.

A threshold has then to be chosen, over (resp. below) which the weighting function is set to 0 (resp. 1), so that it excludes the disturbed area of each image. To find the value of this threshold, one studies the perturbation obtained with a standard DIC algorithm for the smallest angle value presented Fig. 5(a). One defines the disturbed area as the circular region where the perturbation (the standard deviation of the displacement field) is equal to $150 \%$ of the perturbation on an unaffected region. In the case presented Fig. 5(a), the disturbed area radius equals 165 pixels. Considering now the corresponding blurred image Fig. 16(b), the average grey level value on the perimeter of the disturbed area is $69 \%$ of the total dynamic range. To have a conservative threshold, $66 \%$ (2/3) of the dynamic range is chosen and applied thereafter to every weighting function calculation. Fig. 16(c) presents the $p_{j}$ function obtained for the image Fig. 16(a) with this criterion.

$\varphi_{i}$ functions are optional. They may be useful if the shooting with the different lighting conditions are not done exactly with the same point of view, for example 


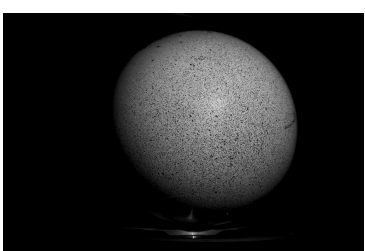

(a)

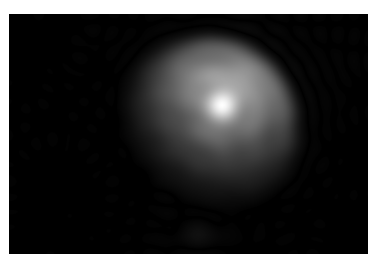

(b)

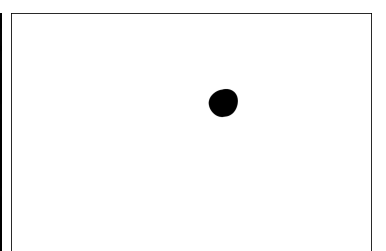

(c)

Fig. 14 Example of $p_{j}$ weighting function calculation: (a) image, (b) blurred image, (c) $p_{j}$ obtained with subsequent values inversion and binarization (threshold $=66 \%$ of the dynamic range).

because the rapid mirror or shutter motions on a digital single lens reflex induce camera displacements. Without the $\varphi_{i}$ functions, a band of high displacement uncertainty appears in the present case at the frontier defined by the weighting function $p_{j}$. The affected elements are the ones crossed by the step of $p_{j}$ function. The $\varphi_{i}$ functions correspond to displacement fields defined on all the image, such as rigid body motions. For this reason, and because the disturbed areas are excluded by the $p_{j}$ functions during the minimization of Eq.3, the influence of the long wave variation of the grey level due to the lightning change is negligible in comparison with the short wave grey level heterogeneity due to the texture, so that the $\beta_{i}$ coefficients are well evaluated. For what we have tested so far, $\varphi_{i}$ functions for uniform camera plane motions were enough to circumvent this problem.

\subsection{Validation}

The same optical setup as in section 2 is used for validation. The observed object is the same $82.5 \mathrm{~mm}$-sphere with the same speckle. For each angle, two light sources $\left(b_{1}\right.$ (as in section 2.2) and $b_{2}$ in Fig. 2) are successively illuminating the sphere, with one image taken for each light source.

The obtained displacement fields $U_{y}$ are presented Fig. 15 for the first and last angle increments $\left(0^{\circ} 20^{\prime}\right.$ and $\left.14^{\circ}\right)$. No disturbed areas are visible. The $p_{j}$ weighting 
functions are presented Fig. $16: P_{1}\left(\right.$ resp. $\left.P_{2}\right)$ corresponds to the images taken with $b_{1}$ (resp. $b_{2}$ ) lightning source in the reference position and with $\alpha_{c}=0^{\circ} 20^{\prime}$ or $14^{\circ} 00^{\prime}$. The excluded areas of each pair are not overlapping, which would result in a displacement field defined by any optical informations on the intersection area. For the large angle value, one clearly sees the two exclusion areas of the reference image and the deformed image for each $p_{j}$, since the disturbed areas have 'moved' about 200 pix. between these two images. The perturbation results are presented on Fig. 17. The perturbation of the different areas is now following the same trend. For area $A 0$ which is never affected by the reflection, the perturbation is lower than with the standard algorithm since two pairs of images are used to assess the displacement field for each angle. For other areas where the masks are used, the perturbation is similar to the one obtained with a standard algorithm using a single image for each angle and an area not affected by a reflection (i.e. $A 0$ on Fig. 7). Area $A 4$, where the reflection is centered in the reference image has also exactly the same trend.

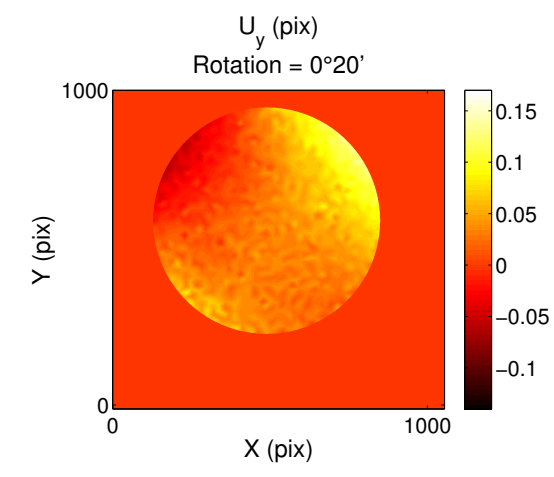

(a)

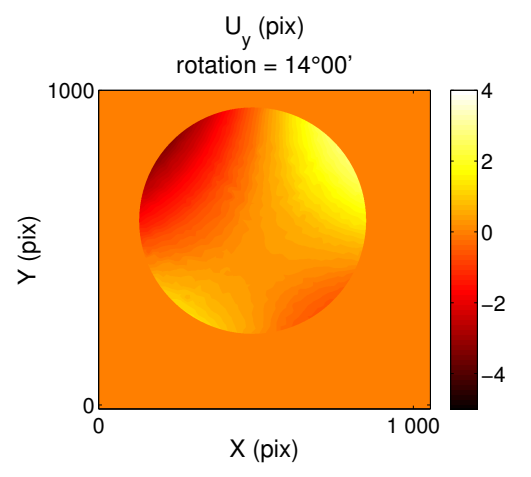

(b)

Fig. 15 Vertical displacement $U_{y}$ measured for a $0^{\circ} 20^{\prime}$ (a) and $14^{\circ}$ (b) rotation with the proposed algorithm.

One thus considers the algorithm as satisfactory for the goals presented previously. 

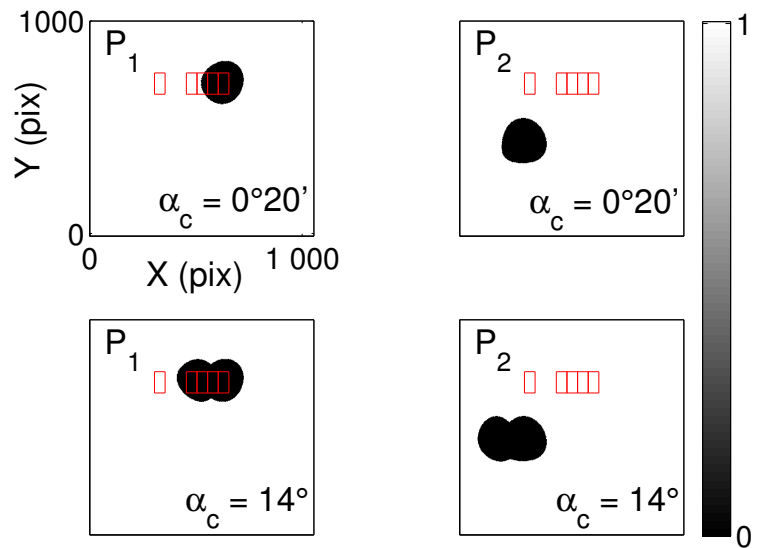

Fig. $16 P_{j}$ functions for the first and the last rotation angle values generated and used by the algorithm. The red rectangles correspond to the areas $A 0$ to $A 4$.

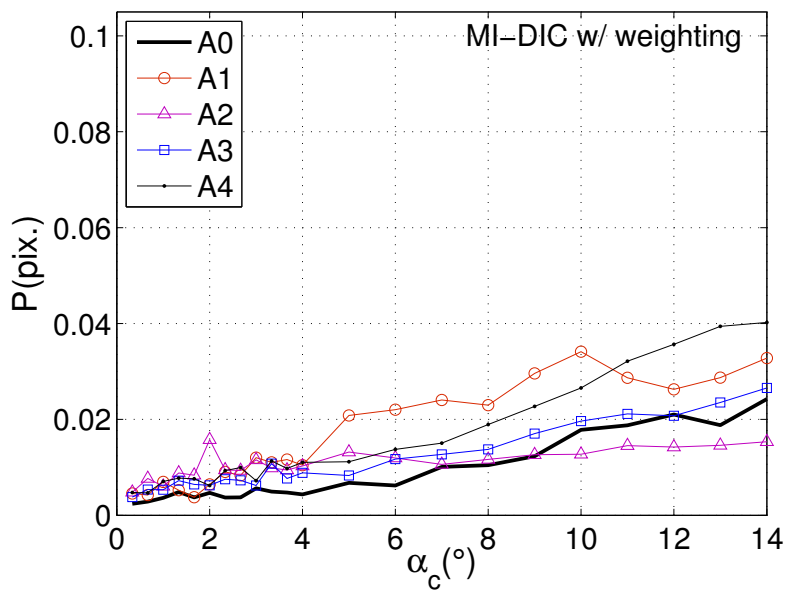

Fig. 17 Perturbation $P$ for the different areas, as a function of the rotation angle, with the proposed algorithm.

\section{Discussion}

Apart from the main result which is the reflection-proofness of the algorithm, three other facts are worth noting. 
First, the perturbation slowly increases for every area. The same phenomenon was observed with the DIC and GLC-DIC algorithms for the $A 0$ area, but to a lesser extent in the latter case: GLC-DIC algorithm is able to partially compensate reflection problems for large angles. This proves that for such important rotation amplitudes, the texture evolution does not strictly satisfy the optical flow conservation assumption. A grey level correction improves the results, but even for GLC-DIC algorithm, the perturbation slightly rises in areas unaffected by the reflection. Based on the speckle study presented in section 2.4 - emphasizing important normal variations - a reasonable hypothesis is that the texture evolution can not be accurately modeled by a strain and a variation of illumination at the element scale.

Second, the use of several pairs of images to assess a displacement field instead of one will necessarily lead to a lower uncertainty. The camera noise $n(\underline{x})$ was assessed to be a white gaussian one. Since the displacement field is obtained through the summation of the contribution of $k$ pairs of images (here $k=2$ ), the central limit theorem implies that the measurement uncertainty (the standard deviation of the displacement field) is divided by $\sqrt{k}$ (here $\sqrt{2}$ ) in comparison with a standard algorithm using only 1 pair of images. If the perturbation of $A 0$ area is compared on Fig. 7 and Fig. 17, one remarks a ratio slightly higher than $\sqrt{2}$. This small difference may be explained by the size of the areas, chosen small enough to have several of them along the reflection trajectory, but not large enough to be statistically representative.

Third, the choice of the weighting function is here arbitrary. A binary function, excluding a region around the reflection, is a first proposition and may be improved. Theses functions were not specifically chosen to be optimal in term of displacement noise reduction. However one notes that even such a simple function leads to a tremendous improvement of the perturbation without any noticeable disturbance at the sharp frontier of the exclusion region. 


\section{Conclusion}

The goal of this work was first to assess the displacement uncertainty due to the presence of a reflection when a DIC algorithm is used at the macroscopic (millimeter, centimeter) scale. The proposed protocol has revealed that the local displacement uncertainty rises up to 5 times the usual one when a reflection occurs. In the case of a DIC algorithm with grey level correction, this increase is less important for large rotation angle values $\left(>2^{\circ}\right)$ but nearly unchanged for small ones. The point source lightnings that were used are of course more critical than more homogeneous ones, but the observed object has a rather soft curvature, so that the obtained results are considered representative of usual experiments.

An observation of the topography of the speckle (a black and white paint spray) has shown an important sub-droplet (and thus sub-pixel) roughness that explains the grey level fluctuations at the pixel scale, spoiling the calculation the gradient of the texture.

To circumvent this reflection issue, a new DIC has been proposed. It performs a single minimization with several images where the reflections are located in different regions. For each image, weighting functions are used to 'exclude' the reflection regions from the calculation, while the necessary information is obtained from the other images. Even with non-optimized weighting functions, very good results are obtained: the displacement is no longer disturbed by the reflection, especially for small angle values $\left(<2^{\circ}\right)$ where the DIC algorithm with grey level correction was unable to improve the results.

Eventually, this new technique with multiple images opens the way to new experimental protocols: more practical ones, like using a single, lightweight, mobile, lightning equipment to illuminate large objects that are submitted to slow evolution (building, ...), or more efficient ones, like using several constant lightnings with different colors and an RGB sensor to capture fast phenomena without reflection issues. 


\section{Acknowledgments}

The authors thank the research team Eikology of the LMT-Cachan laboratory for the helpful discussion on the subject, Y. Quinsat (LURPA, ENS Cachan / CNRS EA 1385) for his help on the confocal chromatic measurement and C. Galetta and

S. Mottola (Master 1 students) for their preliminary work with the authors on the reflection influence on DIC.

\section{References}

1. M. Grédiac, F. Hild, Full-Field Measurements and Identification in Solid Mechanics, M. Grédiac, F. Hild (ed.), ISTE/Wiley (2012).

2. M.A. Sutton,W.J. Wolters, W.H. Peters, W.F Ranson, S.R. McNeill, Determination of displacements using an improved digital correlation method. Image Vis Comput 1(3) 133139 (1983).

3. S. Yoneyama, A. Kitagawa, S. Iwata, K. Tani, H. Kikuta, Bridge deflection measurement using digital image correlation, Experimental Techniques, 31(1) 34-40 (2007).

4. P. Berring, H.W. Knudsen, Torsional performance of large wind turbine blades experimental and numerical analysis. Masters thesis, Department of Mechanical Engineering, Technical University of Denmark (2006).

5. B. Beaubier, J.E. Dufour, F. Hild, S. Roux, S. Lavernhe, K., Lavernhe-Taillard, CAD-Based Calibration and Shape Measurement with StereoDIC - Principle and Application on Test and Industrial Parts, Exp. Mech., 54(3), 329-341 (2014).

6. B. M. B. Grant, H. J. Stone, P. J. Withers, M Preuss, High-temperature strain field measurement using digital image correlation, The Journal of Strain Analysis for Engineering Design 44, 263-271 (2009).

7. J.-H. Kim, A. Serpantié, F. Barlat, F. Pierron, M.-G. Lee, Characterization of the postnecking strain hardening behavior using the virtual fields method. International Journal of Solids and Structures, 50(24), 3829-3842 (2013).

8. J. Sedgewick, Scientific Imaging with Photoshop: Methods, Measurement, and Output, Chap. 6, Peachpit Press, (2010). 
9. G. Babaloukas, N. Tentolouris, S. Liatis, A. Sklavounou, D. Perrea, Evaluation of three methods for retrospective correction of vignetting on medical microscopy images utilizing two open source software tools, Journal of Microscopy, 244(3), 320-324 (2011).

10. J. Gómez-Sanchis, E. Moltó, G. Camps-Valls, L. Gómez-Chova, N. Aleixos, J. Blasco, Correction of the effects of the light source on spherical objects. An application to the analysis of hyperspectral images of citrus fruits, Journal of Food Engineering, 85, 191200 (2008).

11. M. Takeda and K. Mutoh, Fourier transform profilometry for the automatic measurement of 3-D object shapes. Applied Optics 22(24), 3977-3982 (1983).

12. M. Bornert, F. Brémand, P. Doumalin, J.-C. Dupré, M. Fazzini, M. Grédiac, F. Hild, S. Mistou, J. Molimard, J.-J. Orteu, L. Robert, Y. Surrel, P. Vacher, B. Wattrisse, Assessment of digital image correlation measurement errors: methodol- ogy and results, Exp. Mech. 49, 353-370 (2009).

13. M.A. Sutton, J.-J. Orteu, H. Schreier, Image correlation for shape, motion and deformation measurements: basic concepts, theory and applications. New York: Springer (2009).

14. G. Besnard, H. Leclerc, S. Roux, F. Hild, Analysis of Image Series through Digital Image Correlation, Jal of Strain Analysis for Engineering Design, 47(4), 214-228 (2012).

15. A. Maynadier, M. Poncelet, K. Lavernhe-Taillard, S. Roux, One-Shot measurement of thermal and Kinematic fields: InfraRed Image Correlation (IRIC), Experimental Mechanics. 52(3), 241-255 (2012).

16. G. Besnard, F. Hild, J.-M. Lagrange, P. Martinuzzi, S. Roux, Analysis of necking in high speed experiments by stereocorrelation, International Journal of Impact Engineering, 49, 179-191 (2012).

17. M.A. Sutton, S.R. McNeill, J.D. Helm, Y.J. Chao, Advances in two-dimensional and three-dimensional computer vision. In: Rastogi PK (ed) Photomechanics. Springer, Berlin Heidelberg New York, 323-372 (2000).

18. G. Besnard, F. Hild, S. Roux, "Finite-element" displacement fields analysis from digital images: Application to Portevin-Le Châtelier bands, Experimental Mechanics 46(6), 789-804 (2006).

19. H. Leclerc, J.N. Perie, S. Roux, F. Hild, Integrated Digital Image Correlation for the Identification of Mechanical Properties, MIRAGE 2009 - 4th International Conference on Computer Vision/Computer Graphics Collaboration Techniques, Vol 5496, 161-171 (2009). 